\title{
Vorreiter in der Versorgung Herzkranker
}

\author{
Deutschlandweit erste Wachstation für Patienten mit schwerer Herzschwäche
}

Das Universitätsklinikum Heidelberg hat die deutschlandweit erste Wachstation ausschließlich für Patienten mit fortgeschrittener Herzschwäche eröffnet. Die schwer herzkranken Patienten, die z. B. unter akuter Herzmuskelentzündung leiden, sich von einem Infarkt erholen müssen oder auf ein Spenderherz warten, können in der "Advanced Heart Failure Unit“ (AHFU) nun intensiver betreut werden als bisher. Dazu tragen ein speziell zusammengesetztes Behandlungsteam, modernste technische Ausstattung sowie die enge Zusammenarbeit von Kardiologen, Herzchirurgen und - wenn der Patient ein junger Erwachsener mit angeborenem Herzfehler ist - Kinder-Kardiologen bei.

„Mit dieser Station haben wir die Behandlung von Patienten mit fortgeschrittener Herzinsuffizienz entscheidend weiterentwickelt. Die AHFU hat deutschlandweit Modellcharakter“, erklärt Prof. Dr. Hugo. A. Katus, Ärztlicher Direktor der Abteilung Kardiologie, Angiologie, Pneumologie an der Medizinischen Universitätsklinik Heidelberg, wo die neue Station angesiedelt ist. Die AHFU verfügt über acht Betten, pro Jahr können circa 400 Patienten dort behandelt werden.

\section{Wichtige Rolle für Heidelberger Transplantationszentrum}

Eine wichtige Rolle spielt die neue Station auch für die Heidelberger Transplantationsmedizin: Patienten, deren Zustand sich ohne Aussicht auf Besserung lebensbedrohlich verschlechtert und die auf der Hochdringlichkeits-Liste für ein Spenderherz stehen, können dank engmaschiger Betreuung durch das erfahrene Team und den Einsatz moderner Herzunterstützungssysteme meist gut stabilisiert werden. „Die Patienten befinden sich zum Zeitpunkt der Transplantation in einem besseren körperlichen Zustand als bei Betreuung in nicht spezialisierten Stationen - das erhöht ihre Überlebenschancen“, erklärt Prof. Dr. Matthias Karck, Ärztlicher Direktor der Universitätsklinik für Herzchirurgie Heidelberg. Zudem können nun mehr Hochdringlichkeits-Patienten gleichzeitig versorgt werden. „Die AHFU trägt wesentlich zum Ausbau des Heidelberger Transplantationszentrums im Be-

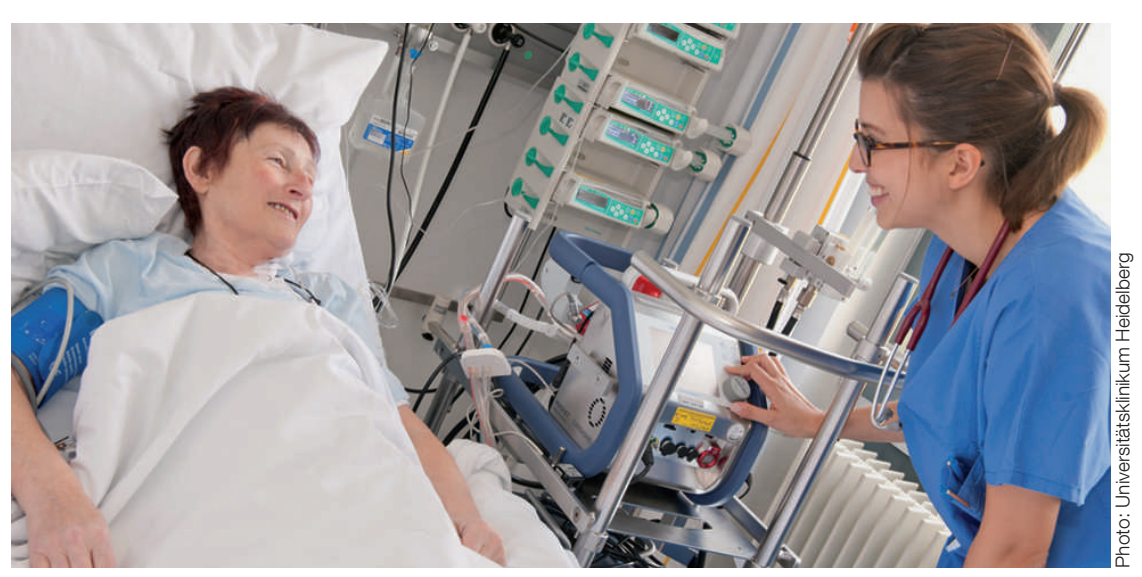

Patientin mit schwerer Herzschwäche auf der neuen Wachstation.

reich der Herztransplantationen bei“, so der Herzchirurg.

Die intensivere und damit bessere Betreuung ist möglich, da die Advanced Heart Failure Unit in der Medizinischen Universitätsklinik ausschließlich auf die Versorgung dieser einen Patientengruppe ausgerichtet ist. Ein spezialisiertes Ärzteund Pflegeteam ist rund um die Uhr vor Ort und kann in schwierigen Situationen souverän und schnell reagieren. „Dank unserer Erfahrung können wir z. B. sehr gut abschätzen, welche Behandlung sich für welchen Patienten am besten eignet, oder ab wann der Patient nicht mehr auf ein Spenderherz warten kann, sondern ein Kunstherz benötigt", erklärt Oberarzt Dr. Philip Raake, Leiter der Station. Er entwickelte einen Therapieplan, der die optimale Versorgung der AHFU-Patienten unter Ausschöpfung aller sinnvollen Therapien gewährleistet. Er kann dabei auf ein weites Spektrum an konventionellen, kathetergestützten und chirurgischen Therapieoptionen zugreifen.

\section{Minimal-invasive \\ Herzunterstützungssysteme immer griffbereit}

An allen acht Plätzen der AHFU können Herzfunktion und Allgemeinzustand der Patienten invasiv und nicht-invasiv überwacht werden. „Unser Ziel ist, die Patienten optimal zu überwachen, damit wir sofort erkennen, wenn das Herz weitere Unterstützung braucht", sagt Raake. In diesem Fall stehen auf der Station mo- dernste minimal-invasive Herzunterstützungssysteme (Assist-Systeme) bereit, die vorrübergehend den Kreislauf aufrecht erhalten, das Herz entlasten und ihm so Gelegenheit geben, sich zu erholen. Sie können aber auch die Zeit bis zu einer Transplantation oder dem Einsatz eines Kunstherzens überbrücken. Ein Beispiel ist eine kleine Katheterpumpe: Sie wird über die Armarterie mittels Katheter in die linke Herzkammer vorgeschoben und pumpt von dort Blut in die Hauptschlagader. „Diese Systeme retten vielen Patienten das Leben, da sonst ihr Kreislauf zum Erliegen käme“, so der Kardiologe.

Die enge Zusammenarbeit mit den Herzchirurgen garantiert einen reibungslosen Übergang bei der Kunstherz-OP oder Herztransplantation. Bei jungen Erwachsenen mit angeborenem Herzfehler verstärken Kinder-Kardiologen aus dem Zentrum für Kinder- und Jugendmedizin das interdisziplinäre Team. „Diese Patienten wurden in der Regel schon seit ihrer Geburt bei uns betreut und behandelt", erklärt Prof. Dr. Matthias Gorenflo, Ärztlicher Direktor der Klinik für Pädiatrische Kardiologie/Angeborene Herzfehler des Zentrums. „Da wir sie als schwerst herzkranke Erwachsene für diese Therapieform nicht mehr in die Kinderklinik aufnehmen können, freut es mich, dass wir die Betreuung auch auf der AHFU gemeinsam mit den Kollegen nahtlos fortsetzen können.“

Quelle: Pressemitteilung Universitätsklinikum Heidelberg

Informationen: www.klinikum.uni-heidelberg.de 